\title{
Active Quenching Technique for YBCO Tapes: Quench Acceleration and Protection
}

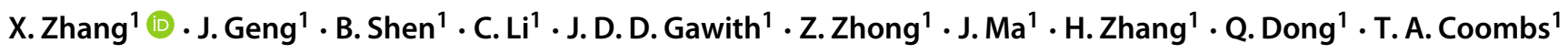

Received: 31 January 2018 / Accepted: 5 February 2018 / Published online: 19 March 2018

(C) The Author(s) 2018

\begin{abstract}
The application of resistive-type superconducting fault current limiters (RSFCLs) in electrical networks is very attractive due to their relative compactness, light weight, and good performance. However, this technology still has drawbacks: asymmetrical quench, uncertain limiting velocity, passive action and incapability of enlarging capacity. Here, we present an active quenching technique which can potentially solve these problems. The quenching process is triggered using highfrequency (HF) AC fields, which are generated by two coupled copper coils attached on both sides of the YBCO sample. Our experiments show that this approach is effective in guaranteeing uniform quench and therefore is expected to be able to significantly extend the service life of the device. Moreover, the quench speed can be considerably increased by the HF field. We find that the performance of acceleration is positively correlated to the transport current, intensity and frequency of the AC field. In addition, a DC magnetic field is added around the sample holder simultaneously with the AC field, to study the field-angular-frequency dependence of the quench time $t\left(B_{\mathrm{AC}} f_{\mathrm{AC}} B_{\mathrm{DC}} \theta_{\mathrm{DC}}\right)$. Experimental results prove that the DC magnetic field can cooperate with the HF AC field to accelerate quench, which means better performance can be produced with lower costs with the two fields acting together. In all, this technique showed outstanding performance regarding quench acceleration and tape protection. We believe the HF-assisted quenching technology has a promising future in current-limiting devices and hope our findings could be helpful for its potential applications.
\end{abstract}

Keywords Active quench $\cdot$ YBCO tapes · Quench protection · Superconducting fault current limiters

\section{Introduction}

With the rapid increase in electrical loads, a considerable percentage of global power systems have been stretched to their designed capacity, leading to a sharp rise of the fault current. Moreover, distributed generation and clean energy sources, such as wind and solar, being added to the already overburdened systems also cause an increase in the fault current level. High fault current levels have severe impacts on power systems. They increase the likelihood of overcurrent damage which may give rise to destruction of electrical facilities or even system blackout [1-4]. The superconducting fault current limiter (SFCL) is a device that can help mitigate the increasing level of fault current

\footnotetext{
X. Zhang

xz326@cam.ac.uk

1 Department of Engineering, University of Cambridge, Cambridge CB3 0FA, UK
}

$[5,6]$. It can also contribute to the performance, stability and efficiency of electricity grids. SFCLs cause no insertion losses during normal operation due to their negligible impedance in the superconducting state. But in the presence of faults, SFCLs are able to quench rapidly to develop impedance so that the short-circuit current can be limited [7]. After faults are cleared, SFCLs then automatically reset to zero resistance and are prepared for the next protective action [8].

The resistive-type SFCL (RSFCL) is one of the most promising choices among all types of SFCLs that have been developed. Due to their structural advantages, RSFCLs can be built very compact and lightweight, and the continuous drop of prices for $2 \mathrm{G}$ coated conductors keeps lowering their manufacturing costs $[9,10]$. There are many RSFCLs that have been built and tested, showing good performance at limiting short-circuit faults [11-16]. However, a few limitations still exist for this technology. First, the critical current properties of high-temperature superconducting (HTS) wires are not uniform in both horizontal and 
perpendicular directions. Therefore, the wires may quench unevenly during faults, leading to regional overheating or even irreversible damage. This problem can be reduced by adding stabilizer into HTS tapes, but insertion of a metal stabilization layer also reduces the normal resistance of RSFCLs [17]. Second, RSFCLs are passive devices since their quench actions are determined by characteristics of the HTS wires. It is therefore not possible to precisely set the triggering current in advance. Third, to guarantee effective current limiting performance, the capacities of RSFCLs have to be designed based on features of present electrical systems, which means considerable upgrade costs are unavoidable with the expansion of power grids. Fourth, the quench velocity cannot be controlled. In cases where faults are not severe, the RSFCLs may not be able to quench fast enough to limit the first peaks of a short-circuit current [18].

We have developed and tested a high-frequency (HF) field-assisted quench approach that helps mitigate these drawbacks of RSFCLs. Two coupled copper coils were fabricated to carry HF AC current with frequency ranging from 10 to $100 \mathrm{kHz}$. By attaching at both sides of the YBCO sample, the two copper coils could provide various HF fields to trigger the active quenching process. Then, the device was placed in a DC magnet, in order to test how the HFassisted quench behaviour of the sample is influenced by external DC fields of different intensities and directions. Using this technique, the tape quenched uniformly with a high velocity, and pre-set of triggering current was also achieved. Moreover, it can be expected that with this design, electrical grid operators can leave certain margin while choosing capacities of RSFCLs. Thus, when the scale of power systems is expanded, they can increase magnitudes of the triggering current instead of replacing all the equipment, so operating costs could be considerably reduced.

This paper is organized as follows. In Section 2, the experimental setup and the controlling logic are presented.
In Section 3, we demonstrate the transient study of active quench initiated by the HF AC field, and analyse factors that have impact on the quench time. Section 4 shows how the DC field influences the HF field-assisted quenching process. Finally, the main conclusions of the paper are summarized in Section 5.

\section{Experimental System}

Figure 1a is a schematic drawing of the entire experimental system, and Fig. 1b shows the installation of the sample holder on the frame of a DC field magnet. The two coupled copper coils wrapped around a superconducting wire are demonstrated in Fig. 1c. The 10-mm-wide YBCO tape used in this experiment was manufactured by Shanghai Superconductor Technology Co., Ltd. (SHSC) [19]. Measurement showed that its self-field critical current was 290 A with a critical electric field criterion $E_{0}=1 \times 10^{-6} \mathrm{~V} / \mathrm{cm}$. The total length of the studied sample was set at $180 \mathrm{~mm}$. Two copper coils made from 18 strands $\emptyset 0.5 \mathrm{~mm}$ wire were coupled together and pressed tightly on the tape surface. Their combined resistance and inductance were $80 \mathrm{~m} \Omega$ and $9.6 \mu \mathrm{H}$ at $77 \mathrm{~K}$, respectively. To minimize the effect of heat generated by the coupled coils, two layers of insulating tape were pasted on the HTS sample side of each copper coil. Then, both the HTS tape and the coupled coils were installed in the sample holder. To guarantee ideal electrical connection, $40 \mathrm{~mm}$ tape from each terminal was surrounded by high-purity indium and clamped by copper plates. The joint resistance was measured to be $0.2 \mathrm{~m} \Omega$. Finally, the sample holder was mounted on the shelf of a 600-mT electromagnet below a high-precision rotary stage, which had a graduation of $1^{\circ}$ and Vernier of $5 \%$

The controlling logic of our experiment can be explained as follows. For the superconducting loop, an Agilent 6680A
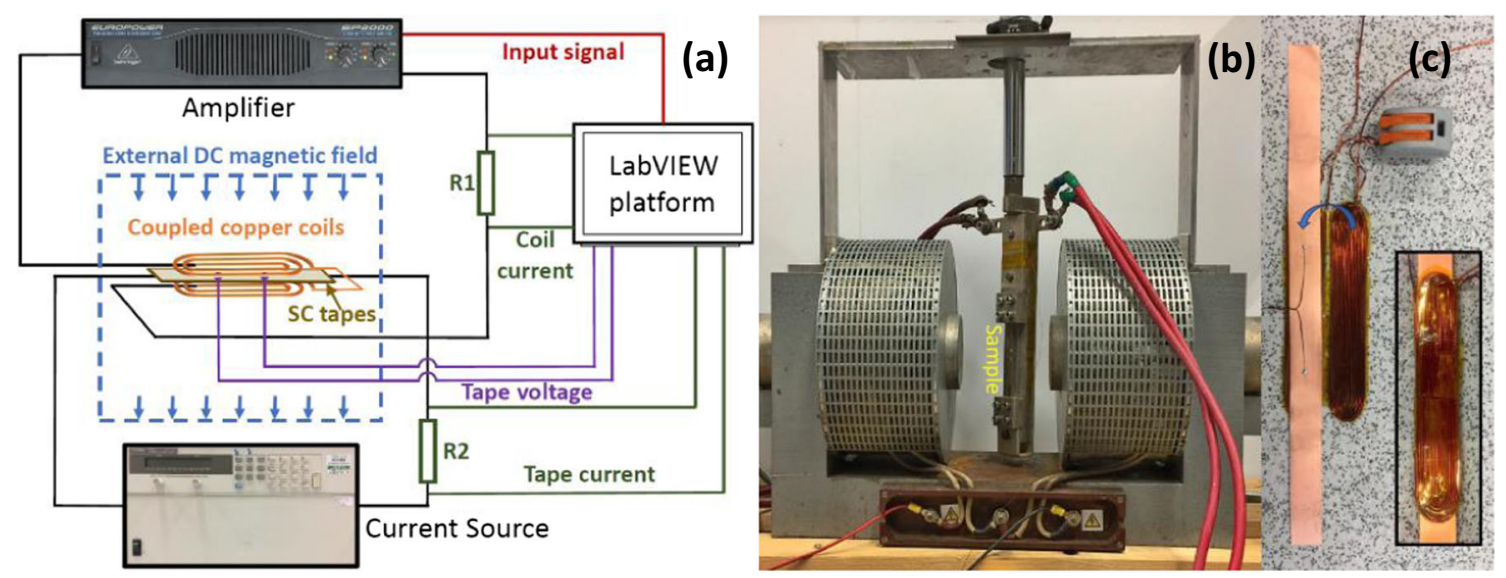

Fig. 1 (Colour online) a Schematic drawing of the experimental system. b Picture of the sample holder in the air gap of the field magnet. $\mathbf{c}$ Picture of the $2 \mathrm{G}$ HTS tape placed in the middle of two coupled copper coils 
was used as DC current source to provide transport current for the HTS wire. To monitor the status of the tape, two voltage taps were soldered on the surface of the YBCO sample $50 \mathrm{~mm}$ apart. The $\mathrm{HF}$ signals injected into the coupled coils were produced by the LabVIEW platform and then amplified to different magnitudes by a Behringer EP4000. With the HF signals, the copper coils can generate an AC magnetic field on the $a b$-plane of the HTS wire. Then, the influence of the HF AC field on the quench behaviour was studied. The experiment was conducted in a liquid nitrogen bath.

\section{Study of Quench Triggered by the HF AC Magnetic Field}

\subsection{Transient Study of the Quench Development}

Many factors such as frequency, intensity and duration of the $\mathrm{HF} \mathrm{AC}$ magnetic field, as well as the transport current carried by the HTS tape, influence the quench characteristics of the YBCO sample [20]. To systematically study the impact of each of them, a series of experiments without the external DC magnetic field was conducted. First, we chose frequency and intensity of the HF AC magnetic field to be $f=50 \mathrm{kHz}$ and $B=20 \mathrm{mT}$, and transport current of the tape $I=150 \mathrm{~A}$. By gradually increasing duration of the applied AC field, the process of quench development of the YBCO tape could be clearly observed.

Figure 2 shows voltage responses of the sample to the HF AC magnetic field with different durations. The distortion in curves was caused by insufficient sampling frequency of our Data Acquisition (DAQ) card. As can be seen from Fig. 2, an alternating voltage between the two voltage taps appeared immediately after applying the HF AC magnetic field. The biased voltage signal was composed of two parts: the induced voltage generated in the voltage measurement circuit and the voltage caused by dynamic resistance (with a DC component) [21]. Figure 2 a showed the result when the AC field was applied for $5 \mathrm{~ms}$. The peak value of biased voltage rose up to $150 \mathrm{mV}$ first and then dropped to zero almost instantaneously at the end of the AC signal. Then, with the duration of the applied field increased to 9 $\mathrm{ms}$, a noticeable delay in sample recovery was found. As shown in the subfigure of Fig. 2b, it took $4 \mathrm{~ms}$ for the

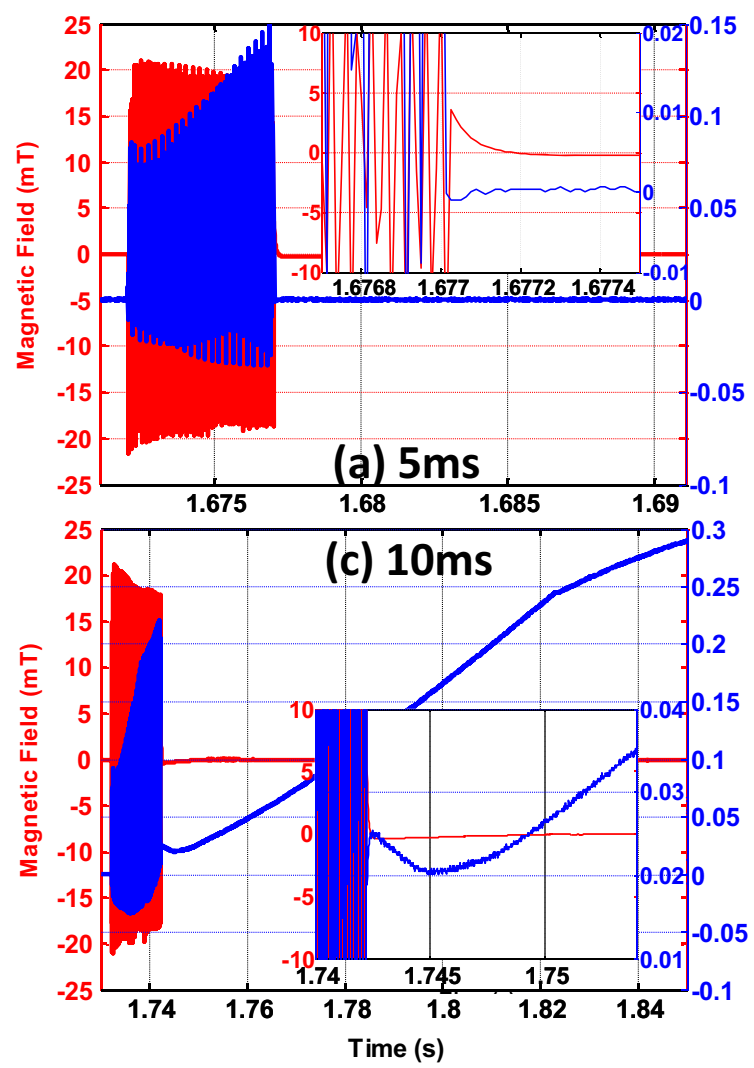

Fig. 2 (Colour online) Dynamic voltage characteristics of the YBCO wire under HF AC magnetic fields of different durations. The YBCO wire was carrying transport current of $150 \mathrm{~A}$. The frequency and
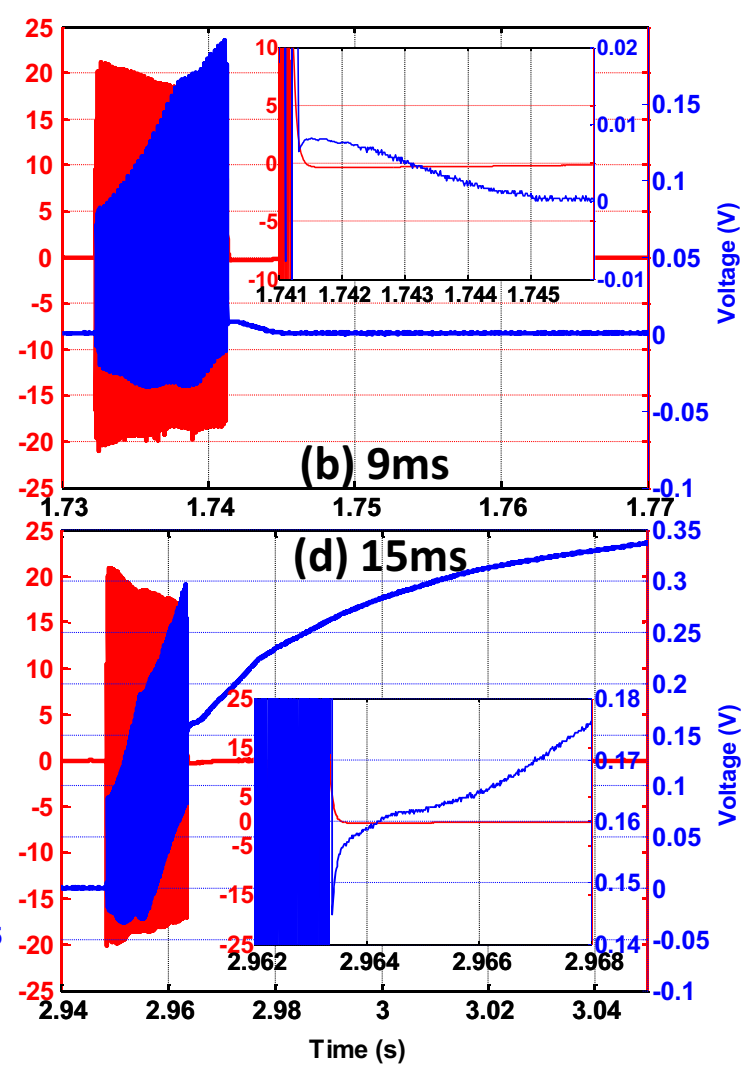

intensity of the HF AC field were $50 \mathrm{kHz}$ and $20 \mathrm{mT}$, respectively. The time lengths of the applied magnetic fields were a $5 \mathrm{~ms}$, b $9 \mathrm{~ms}$, c 10 $\mathrm{ms}$ and $\mathbf{d} 15 \mathrm{~ms}$ 
tape voltage to disappear. However, under the effect of a 10-ms-long AC field, a different behaviour was observed. The sample tended to recover without the applied field, but its voltage increased rapidly after a small drop, and finally led to quench of the entire tape (Fig. 2c). This result implies that the conductor was already in an unstable state due to influence of the HF AC signal. After termination of the applied field, the heat generation rate inside the YBCO wire was still higher than the heat dissipation rate; therefore, quench became unavoidable due to the snowball effect. Figure $2 \mathrm{~d}$ demonstrates that when the $\mathrm{AC}$ field was further lengthened, the peak-to-peak value of the tape voltage does not increase anymore, but the magnitude of the DC component keeps growing constantly, resulting in a faster quench at the end of the applied field.

The quench of the 2G HTS tape was mainly caused by its thermal instability. Under the combined effect of the applied HF AC field and the transport current, several sources contributed to the heat generation and the corresponding temperature rise. First, the change in the external magnetic field generated a loss inside the superconductor, which can be considered as dynamic resistance [21-23]:

$R_{\text {dyn }}=\frac{2 a f L}{I_{\mathrm{c} 0}}\left(B_{a}+\frac{B_{a}^{2}}{B_{0}}\right)$

where $a$ is the width of the sample, $f$ is the frequency of the applied AC magnetic field, and $L$ is the interaction length of the AC field with the YBCO tape, which refers to the length of the coupled coils in our experiment. $I_{\mathrm{c} 0}$ describes the critical current of the studied sample, and $B_{0}$ is the empirical constant of the magnetic field used in the Kim Model.

Although the copper coils lost $\sim 80 \%$ of their room temperature resistance when immersed in the liquid nitrogen bath, the heat transferred from the coils to the sample still cannot be neglected. In addition, AC magnetization loss in the superconductor contributed to the heat generation, and the copper stabilizer of the sample also generates nonnegligible eddy-current loss under the HF AC magnetic field [24]. These sources acting together caused temperature rise of the YBCO tape. After enough heat is accumulated, the critical current of the tape is reduced to near the transport current. Then, the sample enters the flux flow state and starts to generate heat because of the emerging flux flow resistance. This leads to an increase of temperature at a higher rate [25]. The heat dissipation of the YBCO tape was mainly due to heat transfer between the tape surface and liquid nitrogen. When heat generation was too high, the liquid nitrogen vaporized. Since the intervals between YBCO sample and copper coils were very narrow, the nitrogen bubbles got trapped, which significantly weakened the heat dissipation capability. This positive feedback considerably accelerated the heat accumulation process and finally gave rise to quench of the entire tape.

\subsection{Study on Influence Factors of the Quench Time}

As stated in Section 3.1, there were three factors which influenced the quench time of the sample: the magnitude of the transport current, and the frequency and the intensity of the applied HF AC magnetic field. To investigate their relationship with the quench time, a series of experiments was conducted with the following parameters: transport current of 50,100, 150 and $200 \mathrm{~A}$; frequency of the $\mathrm{AC}$ field at 10, 20, 50 and $100 \mathrm{kHz}$; and intensity of the $\mathrm{AC}$ field ranging from 10 to $50 \mathrm{mT}$. Due to capacity constraints of the amplifier, a high intensity of the AC field gradually became unachievable with an increase of the field frequency. However, the experiments that we were able to perform were very representative and more than enough for the analysis. All results about the quench time are shown in Table 1.

Unlike in [26] where the frequency of the applied magnetic field was more than $100 \mathrm{kHz}$, we found that one

Table 1 Variation of quench time along with change in transport current and applied HF AC magnetic field

\begin{tabular}{|c|c|c|c|c|c|c|c|c|c|c|c|}
\hline & $10 \mathrm{mT}$ & $20 \mathrm{mT}$ & $30 \mathrm{mT}$ & $40 \mathrm{mT}$ & $50 \mathrm{mT}$ & & $10 \mathrm{mT}$ & $20 \mathrm{mT}$ & $30 \mathrm{mT}$ & $40 \mathrm{mT}$ & $50 \mathrm{mT}$ \\
\hline \multicolumn{6}{|c|}{ (a) Transport current, $50 \mathrm{~A}$} & \multicolumn{6}{|c|}{ (b) Transport current, $100 \mathrm{~A}$} \\
\hline $10 \mathrm{kHz}$ & No & $106 \mathrm{~ms}$ & $64 \mathrm{~ms}$ & $41 \mathrm{~ms}$ & $26 \mathrm{~ms}$ & $10 \mathrm{kHz}$ & No & $87 \mathrm{~ms}$ & $32 \mathrm{~ms}$ & $16 \mathrm{~ms}$ & $10 \mathrm{~ms}$ \\
\hline $20 \mathrm{kHz}$ & No & $74 \mathrm{~ms}$ & $40 \mathrm{~ms}$ & $25 \mathrm{~ms}$ & & $20 \mathrm{kHz}$ & No & $36 \mathrm{~ms}$ & $14 \mathrm{~ms}$ & $9 \mathrm{~ms}$ & \\
\hline $50 \mathrm{kHz}$ & No & $52 \mathrm{~ms}$ & & & & $50 \mathrm{kHz}$ & No & $25 \mathrm{~ms}$ & & & \\
\hline $100 \mathrm{kHz}$ & No & & & & & $100 \mathrm{kHz}$ & $197 \mathrm{~ms}$ & & & & \\
\hline \multicolumn{6}{|c|}{ (c) Transport current, $150 \mathrm{~A}$} & \multicolumn{6}{|c|}{ (d) Transport current, $200 \mathrm{~A}$} \\
\hline $10 \mathrm{kHz}$ & No & $27 \mathrm{~ms}$ & $19 \mathrm{~ms}$ & $13 \mathrm{~ms}$ & $6 \mathrm{~ms}$ & $10 \mathrm{kHz}$ & $107 \mathrm{~ms}$ & $13 \mathrm{~ms}$ & $9 \mathrm{~ms}$ & $7 \mathrm{~ms}$ & $4 \mathrm{~ms}$ \\
\hline $20 \mathrm{kHz}$ & $102 \mathrm{~ms}$ & $20 \mathrm{~ms}$ & $10 \mathrm{~ms}$ & $5 \mathrm{~ms}$ & & $20 \mathrm{kHz}$ & $53 \mathrm{~ms}$ & $9 \mathrm{~ms}$ & $5 \mathrm{~ms}$ & $4 \mathrm{~ms}$ & \\
\hline $50 \mathrm{kHz}$ & $84 \mathrm{~ms}$ & $11 \mathrm{~ms}$ & & & & $50 \mathrm{kHz}$ & $44 \mathrm{~ms}$ & $6 \mathrm{~ms}$ & & & \\
\hline $100 \mathrm{kHz}$ & $72 \mathrm{~ms}$ & & & & & $100 \mathrm{kHz}$ & $38 \mathrm{~ms}$ & & & & \\
\hline
\end{tabular}


tenth of their predicted value was high enough to trigger the quench process, as can be seen from each sub-table. This finding is important for the promotion of this technology. Moreover, the time required for the YBCO tape to quench kept decreasing when the frequency or the intensity of the applied magnetic field was increased. For instance, Table 1d shows that while the frequency remained at $10 \mathrm{kHz}$ and the field intensity was increased from 10 to $50 \mathrm{mT}$, the quench time of the sample dropped significantly from 107 to $4 \mathrm{~ms}$. Similarly, after we increased the frequency of the $10 \mathrm{mT} / 10$ $\mathrm{kHz}$ AC magnetic field to $100 \mathrm{kHz}$, the quench time of the sample dropped from 107 to $38 \mathrm{~ms}$.

The reason can be explained as follows. As we have stated in the previous section, the quench was triggered mainly by dynamic resistance loss, magnetization loss, eddy-current loss in the copper stabilizer, flux flow resistance loss and heat transferred from the coupled coils to the sample. Since the dynamic resistance, magnetization loss and eddy-current loss are all in direct proportion to the frequency of the applied field, the heat generation rate rises with an increase in frequency. Therefore, the quenching process is shortened correspondingly. In contrast, these three heat sources and the heat generated in coupled coils are directly proportional to the square of the field intensity. Hence, a more obvious acceleration effect is observed when the field intensity is enhanced.

Under the same applied field, the higher the transport current, the faster the quenching process will be. This happens for two reasons. First, when a high transport current is flowing, less heat is required by the sample to enter flux flow state. Hence, less time will be needed for the YBCO tape to quench. Second, according to Oomen [23], (1) is valid only if the intensity of the applied AC field is higher than the full penetration field of the HTS sample. In other words, the applied field must be stronger than a threshold value, so that it can interact with the transport current carried by the tape. A higher transport current means a lower threshold field for the generation of dynamic resistance, and therefore, the sample can quench faster under the same AC magnetic field. This is also a main reason that in half of the $10 \mathrm{mT}$ cases the magnetic field is not capable of triggering the quenching process: an important heat source is missing since the field intensity is too low to overcome the threshold field.

As suggested by Vyacheslav et al. [26],similar designs could increase the uniformity of quench and therefore increase the service life of HTS wires. This phenomenon was also found during our experiments. Under proper operation, the studied sample quenched more than 150 times but still showed no noticeable degradation. The reason for this is that unprotected quench of a YBCO tape tends to start from defects and then propagates slowly. The low normal zone propagation velocity [27, 28] gives rise to extreme thermal stress near the defects, and then damages or even destroys an entire sample. However, due to the flux flow resistance caused by reduction of critical current and the dynamic resistance, quench processes initiated by an HF magnetic field always develop uniformly. Hence, the imbalance of thermal stress over the tape can be significantly reduced, and the service life can be accordingly extended. This characteristic makes the proposed quench initiation design very useful for the protection of current-limiting devices like SFCLs.

Moreover, according to Table $1 \mathrm{~d}$, when an HF magnetic field of $10 \mathrm{kHz} / 50 \mathrm{mT}$ or $20 \mathrm{kHz} / 40 \mathrm{mT}$ was applied, the quenching process can be triggered in $4 \mathrm{~ms}$. This quench velocity is fast enough for SFCLs, but can still be improved on. Considering the limitation of our equipment, we speculate that further acceleration is possible with an AC field of higher frequency and intensity. Moreover, in our experiment, the transport current is fixed at 200 A. However, fault current flowing through SFCLs will be much higher during system faults, which means uniform flux flow states can be developed faster. With the capability of triggering quench so quickly, even taking into account delays caused by fault detection and HF field generation, SFCLs with application of this technique still can cut faults before their first peaks.

\section{Study of Active Quench Under Various External DC Fields}

Previously, we studied the angular-field dependence of critical current $J_{c}(\theta B)$ of YBCO tapes, which demonstrated that the magnitude and direction of the external DC magnetic field have significant impact on the critical current of studied samples [29]. Combining that conclusion with the present study, we believe that the DC background field might be able to accelerate the HF-assisted quench. If true, this technique will be of great importance for the potential applications of HF-assisted quench in RSFCLs. The reason is that, due to the large amount of HTS conductor in RSFCLs, the copper coils used for generation of AC signals have to be quite long for protecting the full length of the superconductor. It is well known that an increase in wire length leads to growth of resistance and inductance, which means it will be more difficult to generate $\mathrm{HF}$ signals in them. However, if the DC background field can cooperate with the $\mathrm{HF} \mathrm{AC}$ field, then the specification for the AC power supplies can be accordingly reduced. Since providing a static magnetic field is much easier, the entire system will be much cheaper and less technically sophisticated. However, it should be noticed that although the DC magnetic field provides an advantage, it can only assist the $\mathrm{HF}$ AC field instead of replacing it, since the 
DC field is not able to guarantee HTS tapes quenching uniformly.

\subsection{Field-Angular Dependence of the Critical Current of the Studied Sample}

To explore HF-assisted quench of the sample under the external DC magnetic field, firstly, we investigated critical current characteristics of the sample inside various DC fields and orientations. In our previous research, we had generalized a fitting function which was capable of accurately describing $I_{c}(\theta B)$ relationship of 2G HTS tapes [29]:

$I_{\mathrm{c}}(\theta, B)=I_{\mathrm{c} 0}\left[1+\varepsilon_{\theta}\left(\frac{B}{B_{0}}\right)^{\alpha-\beta}\right.$

with

$\varepsilon_{\theta}=\sqrt{\gamma^{-2} \sin ^{2}(\theta)+\cos ^{2}(\theta)}$

where $I_{\mathrm{c} 0}=I_{\mathrm{c}}(0, \theta)$ is the critical current in self-field, $B$ is the norm of the magnetic field intensity and $B_{0}$ and $\beta$ are empirical parameters proposed by Kim [30]. In addition, a variable $\alpha$ is introduced to make this fitting approach more general and more accurate. The applied field was also scaled by $\varepsilon_{\theta}$, a function formed based upon the electron mass anisotropy ratio of the material $\gamma^{2}=m_{c}^{*} / m_{a b}^{*}$ [31].

For validation of (2), in total, we studied ten YBCO tapes manufactured by different companies which had different structures. The precise fitting results of all samples convincingly showed the universality of the approach we proposed. Using this method, the magnetic-angular dependence of the critical current of the sample we used in this study was measured and fitted, as plotted in Fig. 3.

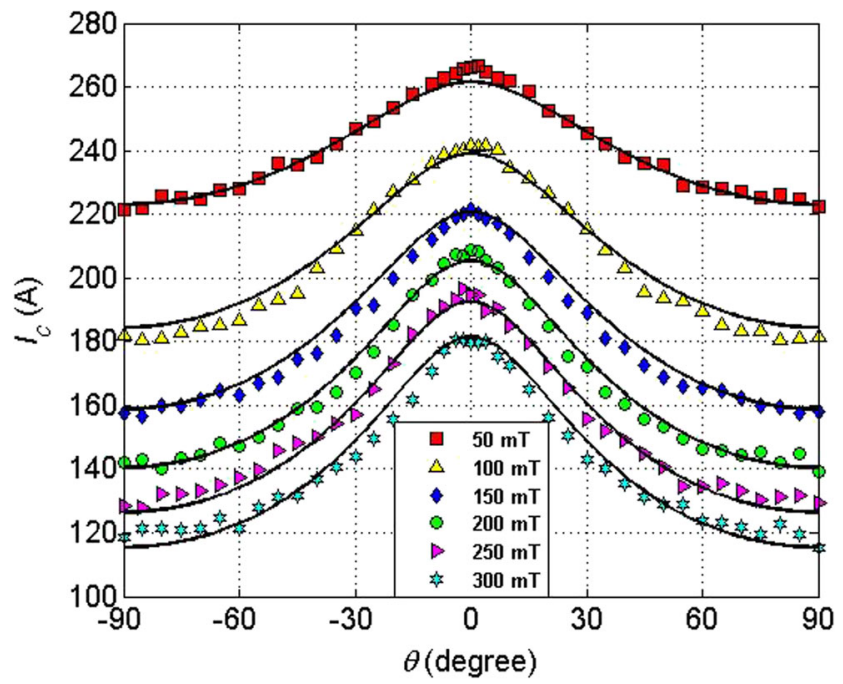

Fig. 3 (Colour online) In-field magnetic-angular dependence of the studied sample: experimental data (symbols) and fitting curves (solid lines). Experimental measurements at less important angles were not plotted for clarity
It can be seen from Fig. 3 that the sample exhibited peaks when the external magnetic field was parallel to the film surfaces $(B / / a b)$ and valleys while the DC field was parallel to the $c$-axis $(B \perp a b)$. In the extreme condition of our experiment, e.g. when the sample was placed in a 300 mT DC background field perpendicular to the $a b$-plane, the critical current decreased from 290 to $120 \mathrm{~A}$. When the $300 \mathrm{mT}$ magnetic field was parallel to the $a b$-plane, it still caused $a \sim 40 \%$ reduction in the critical current (to $180 \mathrm{~A})$. Based on the general fitting approach expressed by (2) and (3), the in-field critical current characteristics of the YBCO tape can be described as $I_{\mathrm{c}}(\theta, B)=290 \times$ $[1+0.35 \times(B / 110)]^{-0.7}$. This fitting result is very precise since the mean absolute percentage deviation is only $1.28 \%$.

\subsection{Relationship Between the Quench Time and the Intensity of the DC Background Field}

Two aspects of the DC magnetic field influence quench behaviour of HTS tapes, namely the intensity and the angle. To investigate the relationship between quench time and field intensity, the DC field was set to be perpendicular to the $a b$-plane of the sample. Then, we fixed transport current at $100 \mathrm{~A}$ and the HF AC field intensity at $10 \mathrm{mT}$, which left two variables in the experiment: the frequency of the HF AC field and the magnitude of the DC field. The quench times we obtained with this setup are plotted in Fig. 4.

As can be seen from Fig. 4, while the DC magnetic field remained unchanged, the increase of the frequency of the HF AC field always reduces the quench time. In addition, the quench process triggered by the same HF

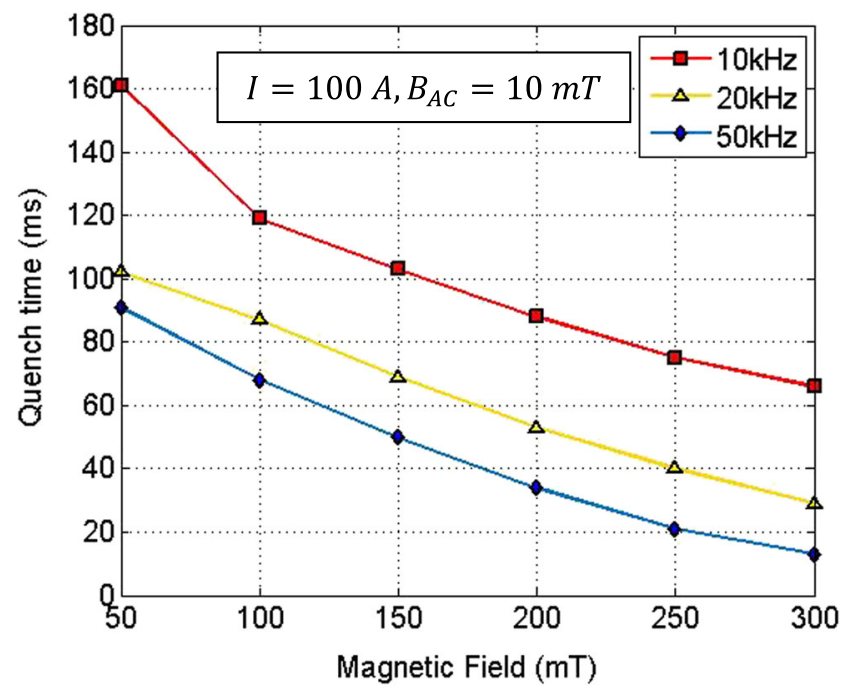

Fig. 4 (Colour online) Reduction in quench time caused by the increase of DC field intensity and the HF AC field frequency. The transport current remained at $100 \mathrm{~A}$, and the intensity of the $\mathrm{HF} \mathrm{AC}$ field was set at $10 \mathrm{mT}$. The direction of the DC field was always perpendicular to the sample surface 
signal accelerated almost linearly when the DC background field became stronger. The reason for the first observation was previously stated in Section 3.2, that the heat generation rate grows along with the rise in the AC field frequency. The second phenomenon was mainly because the existence of the DC magnetic field causes a drop in the critical current of the studied sample, and a lower critical current means faster heat generation and faster entrance to the flux flow state.

According to Table $1 \mathrm{~b}$, if the frequency is lower than $100 \mathrm{kHz}$, an HF AC field of $10 \mathrm{mT}$ does not quench a sample carrying transport current of $100 \mathrm{~A}$. However, under external DC magnetic fields, quench of the sample could always be accomplished, as demonstrated in Fig. 4. To be more specific, with a 300-mT DC background field, the $10 \mathrm{mT}, 10 / 20 / 50 \mathrm{kHz} \mathrm{HF}$ AC signals triggered quench processes within 67/29/13 ms. These durations were shorter than that achieved using $20 \mathrm{mT}, 10 / 20 / 50 \mathrm{kHz}$ HF signals without assistance of DC fields, which was 87,36 and 25 $\mathrm{ms}$, respectively. This shows that a DC field can accelerate the HF-assisted quench. In other words, with the same performance requirement, applying DC background fields can ease demands for AC power sources, and therefore significantly reduce the cost of the system.

Then, to see how the transport current influences the quench time, the sample was placed under different DC background fields while carrying different currents. The AC field was set to be $10 \mathrm{kHz} / 20 \mathrm{mT}$. This group of experimental results was gathered in Fig. 5.

As shown in Fig. 5, when the magnitude of the transport current changed, the effects of quench acceleration caused by the increase of the DC field intensity became markedly different. To begin with, the transport current of the

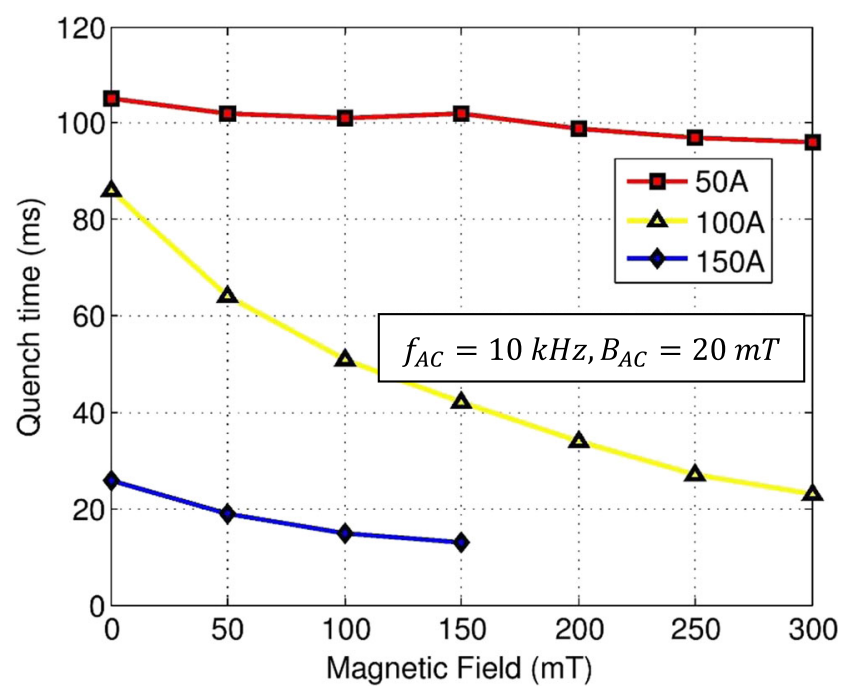

Fig. 5 (Colour online) Quench time of the sample when quench processes were triggered by a $10 \mathrm{kHz} / 20 \mathrm{mT}$ AC magnetic field. The experiments were conducted when the sample was placed in different DC background fields and carried different transport currents studied sample was set to be 50 A. By enhancing the DC background field from 0 to $300 \mathrm{mT}$, there was only a slight decrease in quench time. This was mainly because the critical current of the sample under the 300-mT DC field was much higher than $50 \mathrm{~A}$, so the heat generation was still small due to a low-energy AC signal and low transport current. By considering the redundant cooling capacity and the boiling-off characteristics of liquid nitrogen, the small amount of excess heat brought by the rise of the DC field is consumed relatively fast. Hence, it barely had any effect on the quench time. However, the situation became different after we increased the transport current to $100 \mathrm{~A}$. Originally, it took the HF AC signal $87 \mathrm{~ms}$ to quench the sample. Under the influence of a 300-mT DC background field, a $74 \%$ reduction of the quench time was observed. The reason is similar to the explanation of the phenomenon shown in Fig. 4. In addition, it can also be noticed that with a higher transport current (150A) the acceleration effect got weaker again with the quench time dropping from 27 to $12 \mathrm{~ms}$. According to these results, we conclude that the performance of adding the DC background field is dependent on the amplitude of the transport current and properties of HF AC signals. Therefore, when designing a quench system for RSFCLs using this technique, the magnitude of both the AC and DC fields should be chosen carefully to achieve optimal performance with the least cost and complexity.

\subsection{Relationship Between the Quench Time and the Angle of the DC Background Field}

We investigated the relationship between the quench characteristics and the angle of the DC magnetic field. The magnitude of the HF AC field was fixed at $10 \mathrm{mT}$, with a frequency of 10,20 or $50 \mathrm{kHz}$. To observe the angular dependence clearly, we set the DC field intensity at 300 $\mathrm{mT}$. The DC field was always perpendicular to the transport current, which is the maximum Lorentz force configuration. The field angle $\theta$ is defined as $0^{\circ}$ while the DC field is parallel to the $a b$-plane of the sample. We measured the quench time for every $5^{\circ}$ near the centre (from -10 to $10^{\circ}$ ) and every $10^{\circ}$ at other angles. The experimental results are shown in Fig. 6.

As can be seen from Fig. 6, no matter the AC field frequency, the quench time was always considerably influenced by the angle between the DC magnetic field and sample surface. The $10-\mathrm{kHz}$ AC signal applied parallel to the $a b$-plane of the sample quenched the tape in $241 \mathrm{~ms}$. However, with a $90^{\circ} \mathrm{AC}$ field, this figure dropped to 76 ms, which means the quench time was reduced by $68 \%$. The curve of quench time triggered by the $20 \mathrm{kHz}$ signal appeared to be flatter than the $10 \mathrm{kHz}$ case, but nearly the same decline proportion $65 \%$ (103 and $35 \mathrm{~ms}$, respectively) 


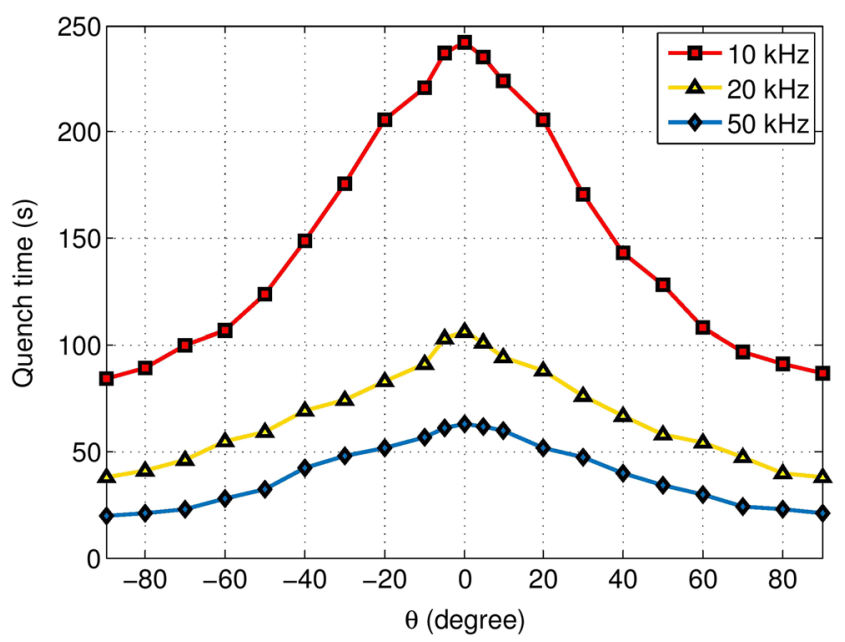

Fig. 6 (Colour online) Angular dependence of quench time under 300 mT DC background field. The sample carried 100 A transport current, and its quench procedures were triggered by a $10 \mathrm{mT} \mathrm{HF}$ AC magnetic field with frequency of 10,20 and $50 \mathrm{kHz}$

was observed. Similar behaviour was found with the $50-\mathrm{kHz}$ scenario. Along with the rotation of AC triggering signal from 0 to $90^{\circ}$, the quench time decreased by $67 \%$ from 66 to $21 \mathrm{~ms}$. It can be concluded that within the studied frequency range, the quench time can be reduced more than $60 \%$ by adjusting the direction of the 300-mT DC field. During the design of potential applications, this should be carefully considered to guarantee optimal performance.

\subsection{Magnetic Field-Angular Dependence of the Quench Time}

In previous subsections, we have separately studied how the quench time is influenced by the intensity and the angle of the DC background field. The next step was to produce a comprehensive figure, which can provide a clearer understanding of DC field intensity dependence and angular dependence of the quench time simultaneously. We used transport current of $100 \mathrm{~A}$ and fixed the HF AC magnetic field to be $10 \mathrm{kHz} / 10 \mathrm{mT}$. For the DC background field of each intensity between 50 and $300 \mathrm{mT}$ (with intervals of $50 \mathrm{mT}$ ), the quench time was recorded with angles ranging from -90 to $90^{\circ}$. This information about the anisotropic field dependence of quench time is plotted in Fig. 7.

Figure 7 demonstrates that applying a DC background field always reduces the quench time regardless of the field intensity. According to Table 1, the tape needed $87 \mathrm{~ms}$ to quench without assistance of the DC field. Under the field of $50 \mathrm{mT}$, this value dropped to $63 \mathrm{~ms}$, showing more than $25 \%$ increase in quench speed. Moreover, with the 300-mT DC field perpendicular to the $a b$-plane of the tape surface, the quench process could be triggered within $24 \mathrm{~ms}$, only

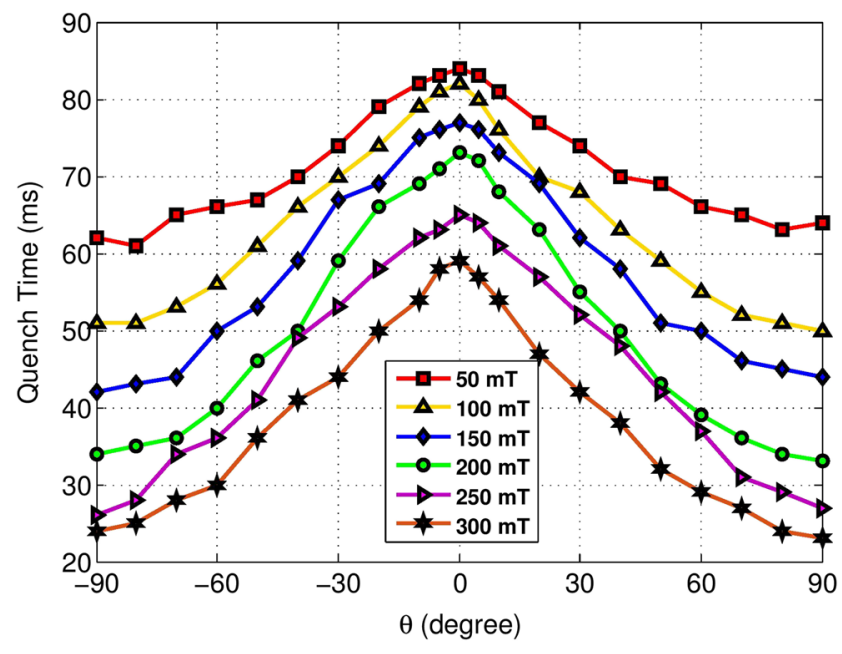

Fig. 7 (Colour online) Magnetic field-angular dependence of quench time of the YBCO sample. The transport current of the tape was $100 \mathrm{~A}$ and the $\mathrm{HF}$ AC field used to trigger quench processes was $10 \mathrm{kHz} / 20$ $\mathrm{mT}$

a quarter of the original figure. In addition, the anisotropy of the quench time was relatively large and kept growing with the increase of DC field intensity. As can be seen from the comparison between Figs. 3 and 7, this phenomenon is in accordance with the $J_{c}(\theta B)$ dependence of the sample. Figure 3 shows that under the DC magnetic field from 50 to $300 \mathrm{mT}$, the percentage deviations between the critical current at 0 and $90^{\circ}$ are $17,25,27,31,35$ and $34 \%$. Correspondingly, the percentage deviations between quench time at 0 and $90^{\circ}$ DC background field are 24, 39, 45, 52, 58 and $58 \%$. The two sets of data are roughly in proportion, but the anisotropy of the quench time is greater. This is mainly because both the dynamic resistance loss and the magnetization loss in the superconductor increase with a drop of the critical current. It becomes easier for the tape to enter the flux flow state, and the sample starts to generate heat due to flux flow resistance. Based on this result, we deduce that with different transport currents and different HF AC signals, the magnetic field-angular dependence of the quench time would follow the same pattern.

\section{Conclusion}

In this paper, we have presented an HF AC-assisted quench study of a YBCO conductor. The development process of the quench triggered by the $\mathrm{AC}$ magnetic field was recorded, and the quench time relationship with the transport current and the AC field was studied. Unlike the deduction given in [26], we found that $\mathrm{AC}$ signals of $10 \mathrm{kHz}$ could trigger quench of the HTS tape. Also, the device proved to be effective at guaranteeing uniform quench of the sample. Our experiments showed that the efficiency of quench was in 
direct proportion to the current carried by the $\mathrm{YBCO}$ wire, the intensity and the frequency of the HF AC magnetic field. Based on these observations, it is concluded that the quench procedures were developed mainly due to dynamic resistance loss, magnetization loss, eddy-current loss in the copper stabilizer, flux flow resistance loss and heat transferred from the coupled coils.

We placed the HTS sample and the coupled copper coils in a DC magnet, to study whether the quench process can be accelerated by the application of the DC magnetic field. We found that with its direction constant, the DC field could effectively reduce the time required for quench, but its performance was dependent on the amplitude of the transport current and characteristics of HF AC signals. In addition, the angle between the DC magnetic field and the tape surface had a huge impact on the quench time, which can be more than $60 \%$. By applying both DC and $\mathrm{AC}$ magnetic fields, the total cost of the device can be considerably reduced. A comprehensive field-angular dependence of the quench time of the studied sample was produced, revealing the same pattern as $J_{c}(\theta B)$ dependence of the tape, but with a greater anisotropy. To summarize, this technique showed outstanding performance regarding quench acceleration and tape protection. We believe the HF-assisted quenching technology has a promising future in current-limiting devices and hope our findings will be helpful for its potential applications.

Acknowledgements Xiuchang Zhang acknowledges a grant from the China Scholarship Council (No. 201408060080).

Open Access This article is distributed under the terms of the Creative Commons Attribution 4.0 International License (http:// creativecommons.org/licenses/by/4.0/), which permits unrestricted use, distribution, and reproduction in any medium, provided you give appropriate credit to the original author(s) and the source, provide a link to the Creative Commons license, and indicate if changes were made.

\section{References}

1. Didier, G., Lévêque, J.: Influence of fault type on the optimal location of superconducting fault current limiter in electrical power grid. Int. J. Electric. Power Energy Syst. 56, 279-285 (2014)

2. Mahmoudian, A., Niasati, M., Khanesar, M.A.: Multi objective optimal allocation of fault current limiters in power system. Int. J. Electric. Power Energy Syst. 85, 1-11 (2017)

3. Zhang, X., Ruiz, H.S., Geng, J., Shen, B., Fu, L., Zhang, H., et al.: Power flow analysis and optimal locations of resistive type superconducting fault current limiters. SpringerPlus 5, 1972 (2016)

4. Zhang, X., Ruiz, H., Geng, J., Coombs, T.: Optimal location and minimum number of superconducting fault current limiters for the protection of power grids. Int. J. Electric. Power Energy Syst. 87, 136-143 (2017)
5. Noe, M., Steurer, M.: High-temperature superconductor fault current limiters: Concepts, applications, and development status. Supercond. Sci. Technol. 20, R15 (2007)

6. Kalinov, A., Voloshin, I., Fisher, L.: SPICE model of hightemperature superconducting tape: Application to resistive faultcurrent limiter. Supercond. Sci. Technol. 30, 054002 (2017)

7. Bonnard, C.-H., Sirois, F., Lacroix, C., Didier, G.: Multi-scale model of resistive-type superconducting fault current limiters based on 2G HTS coated conductors. Supercond. Sci. Technol. 30, 014005 (2016)

8. Chen, Y., Li, S., Sheng, J., Jin, Z., Hong, Z., Gu, J.: Experimental and numerical study of co-ordination of resistivetype superconductor fault current limiter and relay protection. J. Supercond. Novel Magn. 26, 3225-3230 (2013)

9. Smith, A., Oliver, A., Pei, X., Husband, M., Rindfleisch, M.: Experimental testing and modelling of a resistive type superconducting fault current limiter using $\mathrm{MgB} 2$ wire. Supercond. Sci. Technol. 25, 125018 (2012)

10. Šouc, J., Gömöry, F., Vojenčiak, M.: Coated conductor arrangement for reduced $\mathrm{AC}$ losses in a resistive-type superconducting fault current limiter. Supercond. Sci. Technol. 25, 014005 (2011)

11. Bock, J., Breuer, F., Walter, H., Elschner, S., Kleimaier, M., Kreutz, R., et al.: CURL 10: Development and field-test of a 10 $\mathrm{kV} / 10$ MVA resistive current limiter based on bulk MCP-BSCCO 2212. IEEE Trans. Appl. Supercond. 15, 1955-1960 (2005)

12. Neumueller, H.-W., Schmidt, W., Kraemer, H.-P., Otto, A., Maguire, J., Yuan, J., et al.: Development of resistive fault current limiters based on YBCO coated conductors. IEEE Trans. Appl. Supercond. 19, 1950-1955 (2009)

13. Elschner, S., Kudymow, A., Brand, J., Fink, S., Goldacker, W., Grilli, F., et al.: ENSYSTROB-design, manufacturing and test of a 3-phase resistive fault current limiter based on coated conductors for medium voltage application. Physica C: Supercond. Appl. 482, 98-104 (2012)

14. Kim, H.-R., Yang, S.-E., Yu, S.-D., Kim, H., Kim, W.-S., Park, K., et al.: Installation and testing of SFCLs. IEEE Trans. Appl. Supercond. 22, 5602704-5602704 (2012)

15. Chen, Y., Liu, X., Sheng, J., Cai, L., Jin, Z., Gu, J., et al.: Design and application of a superconducting fault current limiter in DC systems. IEEE Trans. Appl. Supercond. 24, 1-5 (2014)

16. Angeli, G., Bocchi, M., Ascade, M., Rossi, V., Valzasina, A., Ravetta, C., et al.: Status of superconducting fault current limiter in Italy: final results from the in-field testing activity and design of the $9 \mathrm{kv} / 15.6$ mva device. IEEE Trans. Appl. Supercond. 26, 1-5 (2016)

17. Kiss, T., Inoue, M., Kuga, T., Ishimaru, M., Egashira, S., Irie, S., et al.: Critical current properties in HTS tapes. Physica C: Supercond. 392, 1053-1062 (2003)

18. Solovyov, V.F., Li, Q.: Application of active quenching of second generation wire for current limiting. Physica C: Supercond. Appl. 519, 130-136 (2015)

19. Shangai Superconductor Technology Co. Ltda. 2G HTS strip. Available: http://shsctec.com/Pages/SpecialProductList.naspxID= $64 \& \mathrm{cl}=\mathrm{e}$

20. Geng, J., Li, C., Coombs, T.A.: A fast AC field controlled impedance in HTS coated conductors: Response speed and electric field value. IEEE Trans. Appl. Supercond. (2017)

21. Mikitik, G.P., Brandt, E.H.: Generation of a dc voltage by an ac magnetic field in type-II superconductors. Phys. Rev. B 64, 092502 (2001)

22. Andrianov, V., Zenkevich, V., Kurguzov, V., Sytchev, V., Ternovskii, F.: Effective resistance of an imperfect type-II superconductor in an oscillating magnetic field. Inst. of Low Temperature, Moscow (1970) 
23. Oomen, M., Rieger, J., Leghissa, M., Ten Haken, B., Ten Kate, H.: Dynamic resistance in a slab-like superconductor with Jc (B) dependence. Supercond. Sci. Technol. 12, 382 (1999)

24. Carr, W.Jr.: AC Loss and Macroscopic Theory of Superconductors. CRC Press (2001)

25. Risse, M., Aikele, M., Doettinger, S., Huebener, R., Tsuei, C., Naito, M.: Dissipation in the superconducting mixed state in the presence of a small oscillatory magnetic-field component. Phys. Rev. B 55, 15191 (1997)

26. Solovyov, V.F., Li, Q.: Fast high-temperature superconductor switch for high current applications. Appl. Phys. Lett. 103, 032603 (2013)

27. Martinez, E., Angurel, L., Pelegrin, J., Xie, Y., Selvamanickam, V.: Thermal stability analysis of YBCO-coated conductors subject to over-currents. Supercond. Sci. Technol. 23, 025011 (2009)
28. Sumption, M., Majoros, M., Susner, M., Lyons, D., Peng, X., Clark, C., et al.: Thermal diffusion and quench propagation in YBCO pancake coils wound with $\mathrm{ZnO}$ andMylar insulations. Supercond. Sci. Technol. 23, 075004 (2010)

29. Zhang, X., Zhong, Z., Ruiz, H., Geng, J., Coombs, T.: General approach for the determination of the magneto-angular dependence of the critical current of YBCO coated conductors. Supercond. Sci. Technol. 30, 025010 (2016)

30. Kim, Y., Hempstead, C., Strnad, A.: Critical persistent currents in hard superconductors. Phys. Rev. Lett. 9, 306 (1962)

31. Harshman, D., Schneemeyer, L., Waszczak, J., Aeppli, G., Cava, R., Batlogg, B., et al.: Magnetic penetration depth in single-crystal Y Ba 2 Cu 3 O 7. Phys. Rev. B 39, 851 (1989) 